\title{
Optimal Performance of Feedback Control Systems with Limited Communication over Noisy Channels
}

\author{
Aditya Mahajan and Demosthenis Teneketzis \\ Department of Electrical Engineering and Computer Science \\ University of Michigan, Ann Arbor, MI 48109-2122, USA \\ Email: \{adityam,teneket\}@eecs.umich.edu
}

\begin{abstract}
A discrete time stochastic feedback control system with a noisy communication channel between the sensor and the controller is considered. The sensor has limited memory. At each time, the sensor transmits encoded symbol over the channel and updates its memory. The controller receives a noisy version of the transmitted symbol, and generates a control action based on all its past observations and actions. This control action action is fed back into the system. At each stage the system incurs an instantaneous cost depending on the state of the plant and the control action. The objective is to choose encoding, memory updating and control strategies to minimize the expected total costs over a finite horizon, or the expected discounted cost over an infinite horizon, or the expected average cost per unit time over an infinite horizon. For each case we obtain a sequential decomposition of the optimization problem. The results are extended to the case when the sensor makes an imperfect observation of the state of the system.
\end{abstract}

\section{INTRODUCTION}

Recent advances in network and communication technologies have led to an increasing interest in networked control systems (NCS) (see the papers in [1]), in particular, the limitations imposed upon feedback control by the presence of a communication channel in the loop. Most researchers have concentrated on stability analysis of the system. The problem of stabilization of a plant with finite data rate feedback was investigated in [2-15]. LQG stability of deterministic and stochastic systems under various communication constraints (rate limited channels, noisy channels with input power constraint, etc.) was considered in [16-22]. Performance limitation in terms of lower bounds on the separation of differential entropy rates was investigated in [23], [24]. However certain applications require performance metrics more general than asymptotic metrics of stability and separation of differential entropy. In this paper we consider the class of additive performance metrics, where the total cost is the sum of costs along the entire path.

In problems with asymptotic performance metrics, transient behavior need not be optimal, thus strong performance bounds can be derived by using asymptotic results from probability theory, information theory and classical control theory. However, in problems with more general performance metrics, transient behavior needs to be optimal. To the best of our knowledge, performance analysis of such problems has not been addressed in the literature. We identify algorithms to obtain optimal strategies; but we have not been able to find expressions for optimal performance or performance bounds.
We consider a discrete-time feedback control system with a communication channel between the sensor and the controller, shown in Figure 1. Such problems arise when the plant and the controller are geographically separated. We are interested in problems in which the sensor has limited resources while the controller has no resource constraint; we model the sensor as an encoder with finite memory, the channel between the sensor and the controller as noisy, and the channel between the controller and the system as noiseless ${ }^{1}$. At each stage the system incurs an instantaneous cost depending on the state of the plant and the control action. The objective is to choose encoding, memory updating and control strategies to minimize the expected total cost over a finite horizon, or expected discounted cost over an infinite horizon, or expected average cost per unit time over an infinite horizon.

The key contribution of this paper is providing a methodology for determining jointly optimal real-time encoding, memory updating and control strategies for feedback control systems with limited communication over noisy channels. The methodology applies to general non-linear stochastic systems, with an arbitrary additive performance criteria.

The remainder of this paper is organized as follows. We formulate the performance analysis of feedback control systems with limited communication over noisy channels as a decentralized stochastic optimization problem. To illustrate the key concepts associated with our solution methodology we first consider in Section II the finite horizon problem, for which we establish structural results of optimal controller and present a methodology for joint optimization of the encoding, memory updating and control strategies. In Section III we extend the methodology to infinite horizon problems. We discuss computational issues for obtaining numerical solution of the dynamic programming algorithms of finite and infinite horizon problems in Section IV The feedback control problem when the encoder has imperfect observation of the state of the plant is considered in Section V We conclude in Section VI

Notation: We use uppercase letters $(X, Y, Z)$ to denote random variables and lowercase letters to denote their realizations $(x, y, z)$. When we represent a function of random variables as a random variable $\left(P_{X_{t}, M_{t}}, P_{X_{t+1}, M_{t}}, P_{X_{t+1}, M_{t}}^{+}\right)$, a tilde above the variable de-

\footnotetext{
${ }^{1}$ In the sequel we show that this assumption does not entail any loss of generality.
} 
notes its realization $\left(\widetilde{P}_{X_{t}, M_{t}}, \widetilde{P}_{X_{t+1}, M_{t}}, \widetilde{P}_{X_{t+1}, M_{t}}^{+}\right)$. When we use Greek letters to represent a random variable $\left(\pi^{c}, \pi^{l}, \pi^{g}\right)$, a tilde above the variable denotes its realization $\left(\tilde{\pi}^{c}, \tilde{\pi}^{l}, \tilde{\pi}^{g}\right)$. We also use the short hand notation of $x^{t}$ to present the sequence $x_{1}, \ldots, x_{t}$ and similar notation for random variables and functions.

\section{The Finite Horizon PROBlem}

\section{A. Problem Formulation}

Fig. 1. Feedback control system with noisy communication

Consider a discrete time feedback control system as shown in Figure 1, which operates for a horizon $T$. The state evolution is given by

$$
X_{t+1}=f\left(X_{t}, U_{t}, W_{t}\right),
$$

where $f$ is the system evolution function. The variables $X_{t}, U_{t}, W_{t}$ denote the state of the system, the control action and the plant disturbance respectively, at time $t$. We assume that all variables are discrete. For all $t, X_{t}$ takes values in $\mathcal{X} \triangleq\{1,2, \ldots,|\mathcal{X}|\}, U_{t}$ takes values in $\mathcal{U} \triangleq\{1,2, \ldots,|\mathcal{U}|\}$ and $W_{t}$ takes values in $\mathcal{W} \triangleq\{1,2, \ldots,|\mathcal{W}|\}$. The initial state $X_{1}$ is a random variable with PMF $P_{X_{1}}$. The random variables $W_{1}, \ldots, W_{T}$ are i.i.d. with PMF $P_{W}$ and are also independent of $X_{1}$.

The sensor, consisting of an encoder and a memory, makes perfect observations of the state of the system. At each time instant $t$, the encoder generates an encoded symbol $Z_{t}$, taking values in $\mathcal{Z} \triangleq\{1, \ldots,|\mathcal{Z}|\}$, as follows

$$
Z_{t}=c_{t}\left(X_{t}, M_{t-1}\right)
$$

where $c_{t}$ is the encoding function at time $t$ and $M_{t-1}$ denotes the content of the sensor's memory at $t-1$. $M_{t}$ takes values in $\mathcal{M} \triangleq\{1, \ldots,|\mathcal{M}|\}$ and is updated according to

$$
M_{t}=l_{t}\left(X_{t}, M_{t-1}\right),
$$

where $l_{t}$ is the memory update function at time $t$. Observe that the sensor has a finite size memory and though it makes perfect observations of the state of the system, it can not store all the past observations. Thus, it does not have perfect recall and at each stage it must selectively shed information.

The encoded symbol $Z_{t}$ is transmitted over a noisy communication channel and a channel output $Y_{t}$ is generated according to

$$
Y_{t}=h\left(Z_{t}, N_{t}\right),
$$

where $h$ is the channel and $N_{t}$ denotes the channel noise. $Y_{t}$ takes values in $\mathcal{Y} \triangleq\{1, \ldots,|\mathcal{Y}|\}$ and $N_{t}$ takes values in $\mathcal{N} \triangleq$ $\{1, \ldots,|\mathcal{N}|\}$. The sequence of random variables $N_{1}, \ldots, N_{T}$ is i.i.d. with given PMF $P_{N} . N_{1}, \ldots, N_{T}$ are also independent of $X_{1}, W_{1}, \ldots, W_{T}$.

The controller observes the channel outputs and generates a control action $U_{t}$ as follows

$$
U_{t}=g_{t}\left(Y^{t}, U^{t-1}\right),
$$

where $g_{t}$ is the control law at time $t$. $U_{t}$ takes values in $\mathcal{U} \triangleq$ $\{1, \ldots,|\mathcal{U}|\}$. A uniformly bounded cost function $\rho: \mathcal{X} \times \mathcal{U} \rightarrow$ $[0, K], K<\infty$ is given. At each $t$, an instantaneous cost $\rho\left(X_{t}, U_{t}\right)$ is incurred.

The collection $\left(\mathcal{X}, \mathcal{W}, \mathcal{M}, \mathcal{Z}, \mathcal{N}, \mathcal{Y}, \mathcal{U}, P_{X_{1}}, P_{W}, P_{N}, f, h\right.$, $\rho, T)$ is called a perfect observation system. The choice of $(c, l, g), c \triangleq\left(c_{1}, \ldots, c_{T}\right), l \triangleq\left(l_{1}, \ldots, l_{T}\right), g \triangleq\left(g_{1}, \ldots, g_{T}\right)$, is called a design.

The performance of a design, quantified by the expected total cost under that design, is given by

$$
\mathcal{J}_{T}(c, l, g) \triangleq \mathbb{E}\left\{\sum_{t=1}^{T} \rho\left(X_{t}, U_{t}\right) \mid c, l, g\right\},
$$

where the expectation in (6) is with respect to a joint measure on $\left(X_{1}, \ldots, X_{T}, U_{1}, \ldots, U_{T}\right)$ generated by $P_{W}, P_{N}, f, h$ and the choice of design $(c, l, g)$. We are interested in the following optimization problem:

Problem 1: Given a perfect observation system $(\mathcal{X}, \mathcal{W}$, $\left.\mathcal{M}, \mathcal{Z}, \mathcal{N}, \mathcal{Y}, \mathcal{U}, P_{X_{1}}, P_{W}, P_{N}, f, h, \rho, T\right)$, choose a design $\left(c^{*}, l^{*}, g^{*}\right)$ such that

$$
\mathcal{J}_{T}\left(c^{*}, l^{*}, g^{*}\right)=\mathcal{J}_{T}^{*} \triangleq \min _{c, l, g \in \mathscr{C}^{T} \times \mathscr{L}^{T} \times \mathscr{G}^{T}} \mathcal{J}_{T}(c, l, g),
$$

where $\mathscr{C}^{T} \triangleq \mathscr{C} \times \cdots \times \mathscr{C}$ (T times), $\mathscr{C}$ is the space of functions from $\mathcal{X} \times \mathcal{M}$ to $\mathcal{Z}, \mathscr{L}^{T} \triangleq \mathscr{L} \times \cdots \times \mathscr{L}$ (T times), $\mathscr{L}$ is the space of functions from $\mathcal{X} \times \mathcal{M}$ to $\mathcal{M}, \mathscr{G}^{T} \triangleq \mathscr{G}_{1} \times \cdots \times \mathscr{G}_{T}$, and $\mathscr{G}_{t}$ is the space of functions from $\mathcal{Y}^{t} \times \mathcal{U}^{t-1}$ to $\mathcal{U}$.

a) Remark: There is no loss of generality in assuming a noiseless feedback channel. Suppose there is noise in the feedback channel, and the input to the system is $\widehat{U}_{t}$ is a noisy version of $U_{t}$ given by

$$
\widehat{U}_{t}=\hat{h}\left(U_{t}, \widehat{N}_{t}\right)
$$

where $\hat{h}$ is the feedback channel and $\widehat{N}_{t}$ denotes the noise in the feedback channel. $\widehat{N}_{1}, \ldots, \widehat{N}_{T}$ is a sequence of independent variables that is also independent of $X_{1}, W_{1} \ldots, W_{T}$ and $N_{1}, \ldots, N_{T}$. This model can be transformed into one equivalent to (1)-(5) by setting

$$
\begin{aligned}
\widehat{W}_{t} & =\left(W_{t}, \widehat{N}_{t}\right), \\
X_{t+1} & =f\left(X_{t}, h\left(U_{t}, \widehat{N}_{t}\right), W_{t}\right) \triangleq \hat{f}\left(X_{t}, U_{t}, \widehat{W}_{t}\right) .
\end{aligned}
$$

Thus, without loss of generality we can assume a noiseless feedback channel.

\section{B. Salient Features of the Problem}

Problem 1 is a decentralized multi-agent stochastic optimization problem. The agents - the sensor and the controllershare a common objective of minimizing the expected total cost. They have access to different (and non-nested) information about underlying state of nature. Furthermore, the actions taken by an agent at any instant of time affects the observations of the other agent at future time instants. Thus the problem is a sequential (in the sense of [25]) dynamic team with strictly non-classical information structure [26]. Dynamic teams are, in general, functional optimization problems having a complex 
interdependence among the decision rules [27]. This interdependence leads to non-convex (in policy space) optimization problems that are hard to solve (see [28] for an example). Identifying an information state sufficient for performance evaluation [29], [30] is a key step in obtaining a sequential decomposition of such problems. To obtain a sequential decomposition of Problem 1] we proceed as follows. First, we derive structural properties of optimal controllers. Using these structural results we transform Problem 1 into an equivalent optimization problem and identify information states for this equivalent problem. This yields a sequential decomposition for Problem 1 along with a dynamic programming algorithm to obtain an optimal design.

\section{Structural Results}

In this section we present structural properties of optimal controllers. For this purpose we define the following.

Definition 1: Let $P_{X_{t}, M_{t}}, P_{X_{t+1}, M_{t}}$ and $P_{X_{t+1}, M_{t}}^{+}$be random vectors defined as follows:

$$
\begin{aligned}
& P_{X_{t}, M_{t}}(x, m) \\
& =\operatorname{Pr}\left(X_{t}=x, M_{t}=m \mid Y^{t}, U^{t-1}, c^{t}, l^{t}, g^{t-1}\right), \\
& P_{X_{t+1}, M_{t}}(x, m) \\
& =\operatorname{Pr}\left(X_{t+1}=x, M_{t}=m \mid Y^{t}, U^{t}, c^{t}, l^{t}, g^{t}\right), \\
& P_{X_{t+1}, M_{t}}^{+}(x, m) \\
& =\operatorname{Pr}\left(X_{t+1}=x, M_{t}=m \mid Y^{t+1}, U^{t}, c^{t+1}, l^{t}, g^{t}\right) .
\end{aligned}
$$

For any particular realization $y^{t}, u^{t-1}$ and arbitrary (but fixed) choice of $c^{t}, l^{t}$ and $g^{t-1}$, the realization of $P_{X_{t}, M_{t}}$, denoted by $\widetilde{P}_{X_{t}, M_{t}}$, is a PMF on $\left(X_{t}, M_{t}\right)$. If $\left(Y^{t}, U^{t-1}\right)$ is a random vector and $c^{t}, l^{t}, g^{t-1}$ are arbitrary (but fixed) functions, then $P_{X_{t}, M_{t}}$ is a random vector belonging to $\mathcal{P}^{\mathcal{X} \times \mathcal{M}}$, the space of PMFs on $\mathcal{X} \times \mathcal{M}$. Similar interpretations hold for $P_{X_{t+1}, M_{t}}$ and $P_{X_{t+1}, M_{t}}$.

These beliefs given by Definition 1 are related as follows:

Lemma 1: For each stage $t$, there exists a deterministic functions $\psi, \phi$, and $\nu$ such that

$$
\begin{aligned}
P_{X_{t+1}, M_{t}} & =\psi\left(P_{X_{t}, M_{t}}, U_{t}\right), \\
P_{X_{t+1}, M_{t}}^{+} & =\phi\left(P_{X_{t+1}, M_{t}}, c_{t+1}\right), \\
P_{X_{t+1}, M_{t+1}} & =\nu\left(P_{X_{t+1}, M_{t}}^{+}, l_{t+1}\right) .
\end{aligned}
$$

Proof: Consider a component of $\widetilde{P}_{X_{t+1}, M_{t}}$, given by (14) at the top of the page.
Now consider

$$
\begin{aligned}
& \operatorname{Pr}\left(X_{t+1}=x, M_{t}=m, U_{t}=u_{t} \mid y^{t}, u^{t-1}, c^{t}, l^{t}, g^{t}\right) \\
& =\sum_{x_{t} \in \mathcal{X}} \operatorname{Pr}\left(X_{t}=x_{t}, M_{t}=m \mid y^{t}, u^{t-1}, c^{t}, l^{t}, g^{t}\right) \\
& \quad \times \operatorname{Pr}\left(U_{t}=u_{t} \mid y^{t}, u^{t-1}, c^{t}, l^{t}, g^{t}, x_{t}, M_{t}=m\right) \\
& \quad \times \operatorname{Pr}\left(X_{t+1}=x \mid x_{t}, M_{t}=m, y^{t}, u^{t}, c^{t}, l^{t}, g^{t}\right) \\
& \stackrel{(a)}{=} \operatorname{Pr}\left(u_{t} \mid y^{t}, l^{t-1}, g_{t}\right) \\
& \times \sum_{x_{t} \in \mathcal{X}}\left[\operatorname{Pr}\left(X_{t}=x_{t}, M_{t}=m \mid y^{t}, u^{t-1}, c^{t}, l^{t}, g^{t-1}\right)\right. \\
& =\operatorname{Pr}\left(u_{t} \mid y^{t}, l^{t-1}, g_{t}\right) \\
& \quad \times \sum_{x_{t} \in \mathcal{X}}\left[\widetilde{P}_{X_{t}, M_{t}}\left(x_{t}, m\right) \operatorname{Pr}\left(X_{t+1}=x \mid x_{t}, u_{t}\right)\right]
\end{aligned}
$$

where $(a)$ follows from (1) and (5). Combine (14) and (18) and cancel $\operatorname{Pr}\left(u_{t} \mid y^{t}, l^{t-1}, g_{t}\right)$ from the numerator and the denominator of (14), giving

$$
\widetilde{P}_{X_{t+1}, M_{t}}=\psi\left(\widetilde{P}_{X_{t}, M_{t}}, u_{t}\right),
$$

where $\psi$ is given by (14) and 18 .

Consider a component of $\widetilde{P}_{X_{t+1}, M_{t}}^{+}$, given by 17 on the top of the page.

Now consider,

$$
\begin{aligned}
\operatorname{Pr} & \left(X_{t+1}=x, M_{t}=m, Y_{t+1}=y_{t+1} \mid y^{t}, u^{t}, c^{t+1}, l^{t}, g^{t}\right) \\
= & \operatorname{Pr}\left(X_{t+1}=x, M_{t}=m \mid y^{t}, u^{t}, c^{t+1}, l^{t}, g^{t}\right) \\
& \times \operatorname{Pr}\left(Y_{t+1}=y_{t+1} \mid y^{t}, u^{t}, c^{t+1},\right. \\
& \left.\quad l^{t}, g^{t}, X_{t+1}=x, M_{t}=m\right) \\
\stackrel{(b)}{=} & \operatorname{Pr}\left(X_{t+1}=x, M_{t}=m \mid y^{t}, u^{t}, c^{t}, l^{t}, g^{t}\right) \\
& \times \operatorname{Pr}\left(Y_{t+1}=y_{t+1} \mid X_{t+1}=x, M_{t}=m, c_{t+1}\right) \\
= & \widetilde{P}_{X_{t+1}, M_{t}}(x, m) \\
& \times \operatorname{Pr}\left(Y_{t+1}=y_{t+1} \mid X_{t+1}=x, M_{t}=m, c_{t+1}\right)
\end{aligned}
$$

where $(b)$ follows from (2) and (4). Combining (17) and 21) we have

$$
\widetilde{P}_{X_{t+1}, M_{t}}^{+}=\phi\left(\widetilde{P}_{X_{t+1}, M_{t}}, c_{t+1}\right),
$$

where $\phi(\cdot)$ is given by 17 and $(21$. 


$$
\widetilde{P}_{X_{t+1}, M_{t}}(x, m)=\frac{\operatorname{Pr}\left(X_{t+1}=x, M_{t}=m, U_{t}=u_{t} \mid y^{t}, u^{t-1}, c^{t}, l^{t}, g^{t}\right)}{\sum_{x^{\prime}, m^{\prime} \in \mathcal{X} \times \mathcal{M}} \operatorname{Pr}\left(X_{t+1}=x^{\prime}, M_{t}=m^{\prime}, U_{t}=u_{t} \mid y^{t}, u^{t-1}, c^{t}, l^{t}, g^{t}\right)} .
$$

$$
\widetilde{P}_{X_{t+1}, M_{t}}^{+}(x, m)=\frac{\operatorname{Pr}\left(X_{t+1}=x, M_{t}=m, Y_{t+1}=y_{t+1} \mid y^{t}, u^{t}, c^{t+1}, l^{t}, g^{t}\right)}{\sum_{x^{\prime}, m^{\prime} \in \mathcal{X} \times \mathcal{M}} \operatorname{Pr}\left(X_{t+1}=x^{\prime}, M_{t}=m^{\prime}, Y_{t+1}=y_{t+1} \mid y^{t}, u^{t}, c^{t+1}, l^{t}, g^{t}\right)}
$$

$$
\begin{aligned}
& \text { Consider a component of } \widetilde{P}_{X_{t+1}, M_{t+1}} \text {, } \\
& \widetilde{P}_{X_{t+1}, M_{t+1}}(x, m) \\
& =\operatorname{Pr}\left(X_{t+1}=x, M_{t+1}=m \mid y^{t+1}, u^{t}, c^{t+1}, l^{t+1}, g^{t}\right) \\
& =\sum_{m_{t} \in \mathcal{M}} \operatorname{Pr}\left(X_{t+1}=x, M_{t}=m_{t} \mid y^{t+1}, u^{t}, c^{t+1}, l^{t+1}, g^{t}\right) \\
& \times \operatorname{Pr}\left(M_{t+1}=m \mid X_{t+1}=x, M_{t}=m_{t}, y^{t+1}, u^{t},\right. \\
& \left.c^{t+1}, l^{t+1}, g^{t}\right) \\
& \stackrel{(c)}{=} \sum_{m_{t} \in \mathcal{M}} \operatorname{Pr}\left(X_{t+1}=x, M_{t}=m_{t} \mid y^{t+1}, u^{t}, c^{t+1}, l^{t}, g^{t}\right) \\
& \times \operatorname{Pr}\left(M_{t+1}=m \mid X_{t+1}=x, M_{t}=m_{t}, l_{t+1}\right) \\
& \times \sum_{m_{t} \in \mathcal{M}} \widetilde{P}_{X_{t+1}, M_{t}}\left(x, m_{t}\right) \mathbb{1}\left[m=l_{t+1}\left(x, m_{t}\right)\right] \\
& \triangleq \nu\left(\widetilde{P}_{X_{t+1}, M_{t}}, l_{t+1}\right),
\end{aligned}
$$

where $(c)$ follows from $(3)$ and $\mathbb{1}[\cdot]$ is the indicator function.

The above relationships between the controller's beliefs lead to the structural results of the optimal controllers.

Theorem 1: Consider Problem 1 for any arbitrary (but fixed) encoding and memory update strategies $c$ and $l$, respectively. Then, without loss of optimality, we can restrict attention to control laws of the form

$$
U_{t}=g_{t}\left(P_{X_{t}, M_{t}}\right) \text {. }
$$

Proof: Equations (14)-16 of Lemma 1 can be combined to obtain

$$
\begin{aligned}
P_{X_{t+1}, M_{t+1}} & =\nu\left(\phi\left(\psi\left(P_{X_{t}, M_{t}}, U_{t}\right), c_{t+1}\right), l_{t+1}\right) \\
& \triangleq \mu\left(P_{X_{t}, M_{t}}, U_{t}, c_{t+1}, l_{t+1}\right) .
\end{aligned}
$$

Thus for any fixed $c$ and $l, P_{X_{t}, M_{t}}$ is a controlled Markov process with control action $U_{t}$. Further, the expected instantaneous cost can be written as

$$
\begin{aligned}
& \mathbb{E}\left\{\rho\left(X_{t}, U_{t}\right) \mid y^{t}, u^{t}, c^{t}, l^{t}, g^{t}\right\} \\
& \quad=\sum_{x_{t}, m_{t} \in \mathcal{X} \times \mathcal{M}} \rho\left(x_{t}, u_{t}\right) P_{X_{t}, M_{t}}\left(x_{t}, m_{t}\right) \\
& \triangleq \hat{\rho}\left(\widetilde{P}_{X_{t}, M_{t}}, u_{t}\right) .
\end{aligned}
$$

There is a subtle technicality in the first step of [26. See [31] for details. Hence we have a perfectly observed stochastic process $\left\{P_{X_{t}, M_{t}}, t=1, \ldots, T\right\}$ with control action $U_{t}$ and instantaneous cost $\hat{\rho}\left(P_{X_{t}, M_{t}}, U_{t}\right)$. From Markov decision theory [30] we know that there is no loss of optimality in restricting attention to control laws of the form (24).
1) Implication of the structural results: Theorem 1 implies that at each stage $t$, without loss of optimality, we can restrict attention to controllers belonging to the family $\mathscr{G}_{s}$ of functions from $\mathcal{P}^{\mathcal{X} \times \mathcal{M}}$ to $\mathcal{U}$. Thus at each stage we can optimize over a fixed (rather than time-varying) domain. Thus Problem 1 is equivalent to the following problem:

Problem 2: Given a perfect observation system $(\mathcal{X}, \mathcal{W}$, $\left.\mathcal{M}, \mathcal{Z}, \mathcal{N}, \mathcal{Y}, \mathcal{U}, P_{X_{1}}, P_{W}, P_{N}, f, h, \rho, T\right)$, choose a design $\left(c^{*}, l^{*}, g^{*}\right)$ that is optimal with respect to the performance criterion of [6, i.e.,

$$
\mathcal{J}_{T}\left(c^{*}, l^{*}, g^{*}\right)=\mathcal{J}_{T}^{*} \triangleq \min _{c, l, g \in \mathscr{C}^{T} \times \mathscr{L}^{T} \times \mathscr{G}_{s}^{T}} \mathcal{J}_{T}(c, l, g),
$$

where $\mathscr{G}_{s}^{T} \triangleq \mathscr{G}_{s} \times \cdots \times \mathscr{G}_{s}(T$ times $)$.

Thus we have an optimization problem in which the action space is not changing with time. In the next section we provide a sequential decomposition of Problem 2

\section{Joint Optimization}

In this section, we identify information states sufficient for performance evaluation of Problem 2 resulting in its sequential decomposition. Problem 2 is equivalent to Problem 1. hence we also obtain a sequential decomposition of Problem 1 The intuition behind our approach is as follows. As mentioned in Section II-B the agents act in a sequential manner. Let $\pi_{t}^{c}, \pi_{t}^{l}, \pi_{t}^{g}$ be the information states of the encoder, memory update and controller respectively. For these to be valid states, they must satisfy the property

$$
\cdots \rightarrow \pi_{t}^{c} \stackrel{c_{t}}{\longrightarrow} \pi_{t}^{l} \stackrel{l_{t}}{\rightarrow} \pi_{t}^{g} \stackrel{g_{t}}{\longrightarrow} \pi_{t+1}^{c} \rightarrow \cdots
$$

that is, at each time instant $t, \pi_{t}^{l}$ can be determined from $\pi_{t}^{c}$ and $c_{t}, \pi_{t}^{g}$ can be determined from $\pi_{t}^{l}$ and $l_{t}$, and $\pi_{t+1}^{c}$ can be determined from $\pi_{t}^{g}$ and $g_{t}$. This ensures that $\pi_{t}^{c}, \pi_{t}^{l}, \pi_{t}^{g}$ are information states in the sense of [30]. However, a system can have more than one information state, and not all of them are sufficient for performance evaluation (see [29]). To be sufficient for performance evaluation, the information states must absorb/summarize the effect of past decision rules on 
the expected future cost, that is,

$$
\begin{aligned}
\mathbb{E}\left\{\sum_{s=t}^{T} \rho\left(X_{s}, U_{s}\right) \mid c, l, g\right\} \\
=\mathbb{E}\left\{\sum_{s=t}^{T} \rho\left(X_{s}, U_{s}\right) \mid \pi_{t}^{c}, c_{t}^{T}, l_{t}^{t}, g_{t}^{T}\right\} \\
=\mathbb{E}\left\{\sum_{s=1}^{T} \rho\left(X_{s}, U_{s}\right) \mid \pi_{t}^{l}, c_{t+1}^{T}, l_{t}^{t}, g_{t}^{T}\right\} \\
=\mathbb{E}\left\{\sum_{s=t}^{T} \rho\left(X_{s}, U_{s}\right) \mid \pi_{t}^{g}, c_{t+1}^{T}, l_{t+1}^{t}, g_{t}^{T}\right\}
\end{aligned}
$$

Furthermore, to extend the results of the finite horizon problem to infinite horizon problems, we want the domain of information states to be time-invariant.

The following information states satisfy the above requirements.

Definition 2: Let $\Pi$ be the space of probability measure on $\mathcal{X} \times \mathcal{M} \times \mathcal{P}^{\mathcal{X} \times \mathcal{M}}$. Define $\pi_{t}^{c}, \pi_{t}^{l}, \pi_{t}^{g}, t=1, \ldots, T$, as follows:

1) $\pi_{t}^{c}=\operatorname{Pr}\left(X_{t}, M_{t-1}, P_{X_{t}, M_{t-1}}\right)$.

2) $\pi_{t}^{l}=\operatorname{Pr}\left(X_{t}, M_{t-1}, P_{X_{t}, M_{t-1}}^{+}\right)$.

3) $\pi_{t}^{g}=\operatorname{Pr}\left(X_{t}, M_{t}, P_{X_{t}, M_{t}}\right)$.

The unconditional PMFs $\pi_{t}^{c}, \pi_{t}^{l}, \pi_{t}^{g}$ defined above are information states sufficient for performance evaluation of Problem 2 Specifically, they satisfy the following properties:

Lemma 2: $\pi_{t}^{c}, \pi_{t}^{l}, \pi_{t}^{g}$ are information states for the encoder, the memory update and the controller respectively, i.e.,

1) there exist linear transformations $Q^{c}\left(c_{t}\right), Q^{l}\left(l_{t}\right)$, and $Q^{g}\left(g_{t}\right)$ such that

$$
\begin{aligned}
\pi_{t}^{l} & =Q^{c}\left(c_{t}\right) \pi_{t}^{c}, \\
\pi_{t}^{g} & =Q^{l}\left(l_{t}\right) \pi_{t}^{l}, \\
\pi_{t+1}^{c} & =Q^{g}\left(g_{t}\right) \pi_{t}^{g} .
\end{aligned}
$$

2) the conditional expected instantaneous cost can be expressed as

$$
\mathbb{E}\left\{\rho\left(X_{t}, U_{t}\right) \mid c^{t}, l^{t}, g^{t}\right\}=\tilde{\rho}\left(\pi_{t}^{g}, g_{t}\right),
$$

where $\tilde{\rho}$ is a deterministic function.

Proof: This follows from Lemma 1 andDefinition 2 See [31] for details.

Using this result the performance criterion of (6) can be rewritten as

$$
\begin{aligned}
\mathbb{E}\left\{\sum_{t=1}^{T} \rho\left(X_{t}, U_{t}\right) \mid c, l, g\right\} & =\sum_{t=1}^{T} \mathbb{E}\left\{\rho\left(X_{t}, U_{t}\right) \mid c^{t}, l^{t}, g^{t}\right\} \\
& \triangleq \sum_{t=1}^{T} \tilde{\rho}\left(\pi_{t}^{g}, g_{t}\right)
\end{aligned}
$$

where the sequence $\left\{\pi_{1}^{g}, \ldots, \pi_{T}^{g}\right\}$ depends on the choice of $(c, l, g)$. Hence, Problem 2 is equivalent to the following deterministic problem:
Problem 3: Consider a deterministic system with states $\pi_{t}^{c}, \pi_{t}^{l}, \pi_{t}^{g}$. The initial state $\pi_{1}^{c}$ is known and the $t \geq 1$, the system evolves as follows,

$$
\begin{aligned}
\pi_{t}^{l} & =Q^{c}\left(c_{t}\right) \pi_{t}^{c}, \\
\pi_{t}^{g} & =Q^{l}\left(l_{t}\right) \pi_{t}^{l}, \\
\pi_{t+1}^{c} & =Q^{g}\left(g_{t}\right) \pi_{t}^{g},
\end{aligned}
$$

where $c_{t}, l_{t}, g_{t}$ belong to $\mathscr{C}, \mathscr{L}, \mathscr{G}_{s}$ respectively and $Q^{c}, Q^{l}, Q^{g}$ are known linear transformations. At time $t$, an instantaneous cost $\tilde{\rho}\left(\pi_{t}^{g}, g_{t}\right)$ is incurred.

The optimization problem is to determine design $(c, l, g)$, where $c \triangleq\left(c_{1}, \ldots, c_{T}\right), l \triangleq\left(l_{1}, \ldots, l_{T}\right)$, and $g \triangleq$ $\left(g_{1}, \ldots, g_{T}\right)$, to minimize the total cost over horizon $T$, i.e.,

$$
\min _{(c, l, g) \in \mathscr{C}^{T} \times \mathscr{L}^{T} \times \mathscr{G}_{s}^{T}} \sum_{t=1}^{T} \tilde{\rho}\left(\pi_{t}^{g}, g_{t}\right)
$$

This is a classical deterministic optimal control problem; optimal functions $\left(c^{*}, l^{*}, g^{*}\right)$ are determined as follows:

Theorem 2: An optimal design $\left(c^{*}, l^{*}, g^{*}\right)$ for Problem 3 (and consequently for Problem 2 and thereby for Problem 1) is given the following nested optimality equations:

$$
V_{T}^{g}\left(\pi^{g}\right)=\inf _{g_{T} \in \mathscr{G}_{s}} \tilde{\rho}\left(\pi^{g}, g_{T}\right)
$$

and for $t=1, \ldots, T$

$$
\begin{aligned}
V_{t}^{c}\left(\pi^{c}\right) & =\min _{c_{l} \in \mathscr{C}} V_{t}^{l}\left(Q^{c}\left(c_{t}\right) \pi^{c}\right), \\
V_{t}^{l}\left(\pi^{l}\right) & =\min _{l_{l} \in \mathscr{L}} V_{t}^{g}\left(Q^{l}\left(l_{t}\right) \pi^{l}\right), \\
V_{t}^{g}\left(\pi^{g}\right) & =\inf _{g_{t} \in \mathscr{G}_{s}} \tilde{\rho}\left(\pi^{g}, g_{t}\right)+V_{t+1}^{c}\left(Q^{g}\left(g_{t}\right) \pi^{g}\right) .
\end{aligned}
$$

The arg min (or arg inf) at each step determines the corresponding optimal design for that stage. Furthermore, the optimal performance is given by

$$
\mathcal{J}_{T}^{*}=V_{1}^{c}\left(\pi_{1}^{c}\right)
$$

Proof: This is a standard result, see [30, Chapter 2].

\section{INFINITE HORIZON PROBLEM}

In this section we extend the model of Section II-A to an infinite horizon $(T \rightarrow \infty)$ using two performance criteria:

1) Expected Discounted Cost where the performance of a design is determined by

$$
\mathcal{J}^{\beta}(c, l, g)=\mathbb{E}\left\{\sum_{t=1}^{\infty} \beta^{t-1} \rho\left(X_{t}, U_{t}\right) \mid c, l, g\right\},
$$

where $0<\beta<1$ is called the discount factor.

2) Average Cost per unit time where the performance of a design is determined by

$$
\overline{\mathcal{J}}(c, l, g)=\limsup _{T \rightarrow \infty} \frac{1}{T} \mathbb{E}\left\{\sum_{t=1}^{T} \rho\left(X_{t}, U_{t}\right) \mid c, l, g\right\} .
$$

We take the limsup rather than lim as for some designs $(c, l, g)$ the limit may not exist. 
Ideally, while implementing a design for infinite horizon problems, we would like to use time-invariant designs. This motivates the following definition.

Definition 3: A design $(c, l, g), c \triangleq\left(c_{1}, c_{2}, \ldots\right), l \triangleq$ $\left(l_{1}, l_{2}, \ldots\right), g \triangleq\left(g_{1}, g_{2}, \ldots\right)$ is called stationary (or timeinvariant) if $c_{1}=c_{2}=\cdots=c, l_{1}=l_{2}=\cdots=l, g_{1}=$ $g_{2}=\cdots=g$.

Due to the dynamic team nature of the problem, it is not immediately clear whether there exist stationary designs that are optimal (or $\varepsilon$-optimal). In this section we show that for the expected discounted cost problem, without loss of optimality, there exist stationary design that are optimal; for the average cost per unit time problem, under certain conditions, there exist stationary designs that are $\varepsilon$-optimal.

\section{A. Expected Discounted Cost Problem}

Consider the infinite horizon problem with expected discounted cost criterion given by (43). For this problem the relations of Lemma 1 hold, hence the structural result of Theorem 1 is valid, and we can restrict attention to encoders belonging to $\mathscr{G}_{s}$. Define $\pi_{t}^{c}, \pi_{t}^{l}, \pi_{t}^{g}$ as in Definition 2 Lemma 2 can be proved as before. The transformations $Q^{c}, Q^{l}, Q^{g}$ and the expected instantaneous cost $\tilde{\rho}$ are the same as in the finite horizon case. Hence, the infinite horizon problem with the expected discounted cost criterion given by (43) is equivalent to Problem 3 with the optimization criterion given by

$$
\mathcal{J}^{\beta}(c, l, g) \triangleq \mathbb{E}\left\{\sum_{t=1}^{\infty} \beta^{t-1} \rho\left(\pi_{t}^{g}, g_{t}\right) \mid c, l, g\right\} .
$$

For this problem we have the following result:

Theorem 3: For the infinite horizon expected discounted cost problem with the performance criterion given by 43], without loss of optimality, one can restrict attention to stationary designs. Specifically, for any optimal design $\left(c^{\prime}, l^{\prime}, g^{\prime}\right)$ there exists a stationary design $\left(c_{0}^{\infty}, l_{0}^{\infty}, g_{0}^{\infty}\right), c_{0}^{\infty} \triangleq\left(c_{0}, c_{0}, \ldots\right)$, $l_{0}^{\infty} \triangleq\left(l_{0}, l_{0}, \ldots\right)$, and $g_{0}^{\infty} \triangleq\left(g_{0}, g_{0}, \ldots\right)$, such that

$$
V\left(\pi_{1}^{c}\right)=\mathcal{J}^{\beta}\left(c_{0}^{\infty}, l_{0}^{\infty}, g_{0}^{\infty}\right)=\mathcal{J}^{\beta}\left(c^{\prime}, l^{\prime}, g^{\prime}\right),
$$

where $V$ is the unique uniformly bounded fixed point of

$V(\pi)=\min _{(c, l, g) \in \mathscr{C} \times \mathscr{L} \times \mathscr{G}_{s}} \widetilde{\rho}(\widehat{Q}(c, l) \pi, g)+\beta V(\widetilde{Q}(c, l, g)(\pi))$,

with

$$
\begin{gathered}
\widehat{Q}(c, l) \triangleq Q^{l}(l) \circ Q^{c}(c), \\
\widetilde{Q}(c, l, g) \triangleq Q^{g}(g) \circ Q^{l}(l) \circ Q^{c}(c),
\end{gathered}
$$

and $\left(c_{0}, l_{0}, g_{0}\right)$ satisfy

$$
V(\pi)=\widetilde{\rho}\left(\widehat{Q}\left(c_{0}, l_{0}\right) \pi, g_{0}\right)+\beta V\left(\widetilde{Q}\left(c_{0}, l_{0}, g_{0}\right)(\pi)\right) .
$$

Proof: See [31].

\section{B. Average Cost per unit time Problem}

Consider the infinite horizon problem with average cost per unit time criterion given by (44). For this problem the relations of Lemma 1 hold, hence the structural result of Theorem 1 is valid, and we can restrict attention to encoders belonging to $\mathscr{G}_{s}$. Define $\pi_{t}^{c}, \pi_{t}^{l}, \pi_{t}^{g}$ as in Definition 2 Lemma 2 can be proved as before. The transformations $Q^{c}, Q^{l}, Q^{g}$ and the expected instantaneous cost $\tilde{\rho}$ are the same as in the finite horizon case. Hence, the infinite horizon expected discounted cost problem is equivalent to Problem 3 with the optimization criterion given by

$$
\overline{\mathcal{J}}(c, l, g) \triangleq \limsup _{T \rightarrow \infty} \frac{1}{T} \mathbb{E}\left\{\sum_{t=1}^{T} \rho\left(\pi_{t}^{g}, g_{t}\right) \mid c, l, g\right\} .
$$

For this problem we have the following result:

Theorem 4: For the infinite horizon average cost per unit time problem with the performance criterion given by [51, assume

(A1) for any $\varepsilon>0$ there exist bounded measurable functions $v(\cdot)$ and $r(\cdot)$ and design $\left(c_{0}, l_{0}, g_{0}\right) \in \mathscr{C} \times \mathscr{L} \times \mathscr{G}_{s}$ such that for all $\pi$,

$$
\begin{aligned}
v(\pi) & =\min _{c, l, g \in \mathscr{C} \times \mathscr{L} \times \mathscr{G}_{s}} v(\widetilde{Q}(c, l, g) \pi) \\
& =v\left(\widetilde{Q}\left(c_{0}, l_{0}, g_{0}\right) \pi\right),
\end{aligned}
$$

and

$$
\begin{gathered}
\min _{c, l, g \in \mathscr{C} \times \mathscr{L} \times \mathscr{G}_{s}} \widetilde{\rho}(\widehat{Q}(c, l) \pi, g)+r(\widetilde{Q}(c, l, g) \pi) \leq \\
\leq v(\pi)+r(\pi) \leq \widetilde{\rho}\left(\widehat{Q}\left(c_{0}, l_{0}\right) \pi, g_{0}\right)+r\left(\widetilde{Q}\left(c_{0}, l_{0}, g_{0}\right) \pi\right)+\varepsilon .
\end{gathered}
$$

Then for any horizon $T$ and any design $\left(c^{\prime}, l^{\prime}, g^{\prime}\right)$ for that horizon, the stationary design $\left(c_{0}^{\infty}, l_{0}^{\infty}, g_{0}^{\infty}\right), c_{0}^{\infty}=\left(c_{0}, c_{0}, \ldots\right)$, $l_{0}^{\infty}=\left(l_{0}, l_{0}, \ldots\right), g_{0}^{\infty}=\left(g_{0}, g_{0}, \ldots\right)$, satisfies

$$
\mathcal{J}_{T}\left(c_{0}^{T}, l_{0}^{T}, g_{0}^{T}\right)=r\left(\pi_{1}^{c}\right)+T v\left(\pi_{1}^{c}\right) \leq \mathcal{J}_{T}\left(c^{\prime}, l^{\prime}, g^{\prime}\right)+\varepsilon,
$$

where $\alpha^{T}=(\alpha, \ldots, \alpha)$ ( $T$ times) for $\alpha=c_{0}, l_{0}, g_{0}$. Further under (A1), 54) is equivalent to

$$
\overline{\mathcal{J}}\left(c_{0}^{\infty}, l_{0}^{\infty}, g_{0}^{\infty}\right)=v\left(\pi_{1}^{c}\right) \leq \underline{\mathcal{J}}\left(c^{\prime \prime}, l^{\prime \prime}, g^{\prime \prime}\right),
$$

where $\left(c^{\prime \prime}, l^{\prime \prime}, g^{\prime \prime}\right)$ is any infinite horizon policy and

$$
\underline{\mathcal{J}}\left(c^{\prime \prime}, l^{\prime \prime}, g^{\prime \prime}\right)=\liminf _{T \rightarrow \infty} \frac{1}{T} \sum_{t=1}^{T} \widetilde{\rho}\left(\widetilde{Q}\left(c_{t}^{\prime \prime}, l_{t}^{\prime \prime}\right) \pi_{t}, g_{t}^{\prime \prime}\right) \text {. }
$$

$$
\text { Proof: See [31]. }
$$

\section{Implication of the Result}

We have shown that there exist optimal stationary designs for the infinite horizon expected discounted cost problem and, under certain conditions, $\varepsilon$-optimal stationary designs for the infinite horizon average cost per unit time problem. This simplifies the off-line optimization problem since we have to choose the best amongst $\mathscr{C} \times \mathscr{L} \times \mathscr{G}_{s}$ stationary strategies, rather than to choose the best amongst $\mathscr{C}^{\infty} \times \mathscr{L}^{\infty} \times \mathscr{G}_{s}^{\infty}$ timevarying strategies. Further, implementing stationary strategies 
involves implementing one function at each agent which is much simpler than implementing a time-varying strategy.

\section{Computational Issues}

The dynamic program of Theorem 2 for joint optimization of encoding, memory updating and control strategies is similar to a dynamic program for partially observed Markov decision problems (POMDP) with uncountable state space and uncountable action space. The information state $\pi_{t}^{c}$ belongs to $\Pi$, the space of probability measures on $\mathcal{X} \times \mathcal{M} \times \mathcal{P}^{\mathcal{X} \times \mathcal{M}}$, which is a subset of probability measures on $\mathbb{R}^{d}$, with $d=|\mathcal{X}| \times|\mathcal{M}|+1$. The action spaces $\mathscr{F}$ and $\mathscr{L}$ are finite while the action space $\mathscr{G}_{S}$ is uncountable. Therefore, in the dynamic program of Theorem 2 the information state belongs to the space of probability measures on a finite dimensional Euclidean space and an uncountable state space. The standard computational techniques for solving such POMDPs can be used to obtain numerical results.

It is the off-line computation of an optimal design that has exponential complexity. The on-line implementation is simple as we need to implement a stationary design.

\section{SENSORS With IMPERFECT OBSERVATIONS}

So far we have assumed that the sensor perfectly observes the state of the system. However in many practical systems, the sensor observations are noisy due to external disturbances and the intrinsic noise in the measurement hardware. In this section we model this scenario and show that noisy observations by the sensor do not alter the nature of the problem. We first consider the finite horizon case.

\section{A. Problem Formulation}

Fig. 2. Feedback control system with noisy communication and imperfect observations.

Consider a discrete time imperfect observation system as shown in Figure 2 which operates for $T$ time steps. The state of the system $X_{t}$ evolves according to (1). The observations $S_{t}$ made by the observer at time $t$ are noisy version of the state of the system and are given by

$$
S_{t}=\hat{h}\left(X_{t}, \widehat{N}_{t}\right)
$$

where $\widehat{N}_{t}$ denotes the observation noise and $\hat{h}$ is the observation channel. $S_{t}$ takes values in $\mathcal{S} \triangleq\{1, \ldots,|\mathcal{S}|\}$ and $\widehat{N}_{t}$ takes values in $\widehat{N} \triangleq\{1, \ldots,|\widehat{N}|\}$. The sequence of random variables $\widehat{N}_{1}, \ldots, \widehat{N}_{T}$ are i.i.d. with PMF $P_{\widehat{N}} . \widehat{N}_{1}, \ldots, \widehat{N}_{T}$ is also independent of $X_{1}, W_{1}, \ldots, W_{T}, N_{1}, \ldots, N_{T}$.

The sensor is modeled as in Section II-A and operates as follows

$$
\begin{aligned}
Z_{t} & =c_{t}\left(S_{t}, M_{t-1}\right), \\
M_{t} & =l_{t}\left(S_{t}, M_{t-1}\right) .
\end{aligned}
$$

All other components of the system (the channel, the controller and the performance measure) are modeled as in Section II-A The collection of $(\mathcal{X}, \mathcal{W}, \widehat{\mathcal{N}}, \mathcal{S}, \mathcal{M}, \mathcal{Z}, \mathcal{N}, \mathcal{Y}, \mathcal{U}$,
$\left.P_{X_{1}}, P_{W}, P_{\widehat{N}}, P_{N}, f, \mu, h, \rho, T\right)$ is called an imperfect observation system. The choice of $(c, l, g), c \triangleq\left(c_{1}, \ldots, c_{T}\right)$, $l \triangleq\left(l_{1}, \ldots, l_{T}\right), g \triangleq\left(g_{1}, \ldots, g_{T}\right)$, is called a design. The performance of a design, quantified by the expected total cost under that design, is given by (6). We are interested in the following optimization problem:

Problem 4: Given an imperfect observation system $(\mathcal{X}$, $\left.\mathcal{W}, \widehat{\mathcal{N}}, \mathcal{S}, \mathcal{M}, \mathcal{Z}, \mathcal{N}, \mathcal{Y}, \mathcal{U}, P_{X_{1}}, P_{W}, P_{\widehat{N}}, P_{N}, f, \mu, h, \rho, T\right)$, choose a design $\left(c^{*}, l^{*}, g^{*}\right)$ such that

$$
\mathcal{J}_{T}\left(c^{*}, l^{*}, g^{*}\right)=\mathcal{J}_{T}^{*} \triangleq \min _{c, l, g \in \mathscr{C}^{T} \times \mathscr{L}^{T} \times \mathscr{G}^{T}} \mathcal{J}_{T}(c, l, g),
$$

where $\mathscr{C}^{T} \triangleq \mathscr{C} \times \cdots \times \mathscr{C}$ (T times), $\mathscr{C}$ is the space of functions from $\mathcal{S} \times \mathcal{M}$ to $\mathcal{Z}, \mathscr{L}^{T} \triangleq \mathscr{L} \times \cdots \times \mathscr{L}$ (T times), $\mathscr{L}$ is the space of functions from $\mathcal{S} \times \mathcal{M}$ to $\mathcal{M}, \mathscr{G}^{T} \triangleq \mathscr{G}_{1} \times \cdots \times \mathscr{G}_{T}$, and $\mathscr{G}_{t}$ is the space of functions from $\mathcal{Y}^{t} \times \mathcal{U}^{t-1}$ to $\mathcal{U}$.

Although in Problem 4 the encoder does not know the state of the plant, the problem is conceptually same as Problem 1 and the solution methodology of Problem 1 works for Problem 4 with very minor changes.

\section{B. Structural Results}

In this section we present structural properties of optimal controllers. For this purpose define the following:

Definition 4: Let $P_{X_{t}, M_{t}}$ and $P_{X_{t+1}, M_{t}}$ be defined as in Definition 2 Define $P_{X_{t+1}, S_{t}, M_{t}}$ as follows:

$$
\begin{aligned}
& P_{X_{t+1}, S_{t}, M_{t}}(x, s, m) \\
& \quad=\operatorname{Pr}\left(X_{t+1}=x, S_{t}=s, M_{t}=m \mid Y^{t}, U^{t}, c^{t+1}, l^{t}\right) .
\end{aligned}
$$

These beliefs are related as follows:

Lemma 3: For each stage $t$, there exists a deterministic functions $\psi, \hat{\phi}$, and $\hat{\nu}$ such that

$$
\begin{aligned}
P_{X_{t+1}, M_{t}} & =\psi\left(P_{X_{t}, M_{t}}, U_{t}\right), \\
P_{X_{t+1}, S_{t}, M_{t}} & =\hat{\phi}\left(P_{X_{t+1}, M_{t}}, c_{t+1}\right), \\
P_{X_{t+1}, M_{t+1}} & =\hat{\nu}\left(P_{X_{t+1}, S_{t}, M_{t}}, l_{t+1}\right) .
\end{aligned}
$$

Proof: This can be proved along the same lines as the proof of Lemma 1

Using the above relationship it can be shown that the structural result of Theorem 1 also hold for Problem 4 Thus, without loss of optimality, we can restrict attention to controllers of the form (24). These structural results imply that we can formulate a problem equivalent to Problem 4 with a time invariant action space.

\section{Joint Optimization}

We follow the philosophy of Section II-D and use the structural results of previous section to obtain a sequential decomposition for Problem 4

Definition 5: Let $\pi_{t}^{c}, \pi_{t}^{l}, \pi_{t}^{g}, t=1, \ldots, T$ be defined as follows:

1) $\pi_{t}^{c}=\operatorname{Pr}\left(X_{t}, M_{t-1}, P_{X_{t}, M_{t-1}}\right)$.

2) $\pi_{t}^{l}=\operatorname{Pr}\left(X_{t}, M_{t-1}, P_{X_{t}, S_{t-1}, M_{t-1}}\right)$.

3) $\pi_{t}^{g}=\operatorname{Pr}\left(X_{t}, M_{t}, P_{X_{t}, M_{t}}\right)$. 
Lemma 2 holds for $\pi_{t}^{c}, \pi_{t}^{l}, \pi_{t}^{g}$ defined above. Thus, the above unconditional PMFs are information states sufficient for performance evaluation of Problem 4 This can be shown along the same lines as the proof of Lemma 2 Hence, Problem 4 is equivalent to a deterministic problem similar to Problem 3 with the transformations $Q^{c}, Q^{l}, Q^{g}$ appropriately defined. The solution of this deterministic problem is given by nested optimality equations similar to Theorem 2 Hence, we obtain a sequential decomposition of Problem 4 Similar results extend to infinite horizon problems using the ideas of Section III

\section{CONCLUSION}

We have presented a methodology for determining jointly optimal encoding and control strategies for feedback control systems with limited communication over noisy channel. The methodology is applicable to finite horizon problems with expected total cost criterion, to infinite horizon problem with expected discounted cost criterion, and to infinite horizon problem with average cost per unit time criterion. We extend this methodology to problem where the encoder/sensor makes imperfect observations of the state of the system. The resulting optimality equations can be viewed as POMDPs where the state space is a real valued vector and the action space is uncountable. Hence traditional method for solving such POMDPs can be used to obtain a solution for feedback control problems with communication constraints.

The methodology presented here can be used to obtain a sequential decomposition of general dynamic team problems with non-classical information structures.

\section{REFERENCES}

[1] IEEE Trans. Automat. Contr: Special Issue on Networked Control Systems, vol. 49, no. 9, Sept. 2004.

[2] D. F. Delchamps, "Stabilizing a linear system with quantized state feedback," IEEE Trans. Automat. Contr., vol. 35, pp. 916-924, Aug. 1990.

[3] W. S. Wong and R. W. Brockett, "Systems with finite communciation bandwidth constraints II: Stabilization with limited information feedback," IEEE Trans. Automat. Contr., vol. 44, pp. 1049-1053, July 1999.

[4] J. Baillieul, "Feedback desings in information based control," in Proc. Workshop in Stochastic Theory and Control, B. Pasik-Duncan, Ed. Springer Verlag, Oct. 2001, pp. 35-27.

[5] _ "Feedback coding for information based control — operating near the data-rate limit," in Proceedings of 41 st IEEE Conference on Decision and Control, Dec. 2002, pp. 3229-3236.

[6] N. Elia and S. K. Mitter, "Stabilization of linear systems with limited information," IEEE Trans. Automat. Contr., vol. 46, pp. 1384-1400, Aug. 2001.

[7] R. W. Brockett and D. Liberzon, "Quantized feedback stabilization of linear systems," IEEE Trans. Automat. Contr., vol. 45, pp. 1279-1289, July 2000

[8] I. R. Peterson and A. V. Savkin, "Multi-rate stabilization of multivariable discrete-time linear systems via a limited capacity communication channel," in Proceedings of 40th IEEE Conference on Decision and Control, 2001, pp. 304-309.

[9] H. Ishii and B. A. Francis, "Quadratic stabilization of sampled-data systems with quantization," Automatica, vol. 39, no. 10, pp. 1793-1800, 2003.

[10] D. Liberzon, "On stabilization of linear systems with limited information," IEEE Trans. Automat. Contr., vol. 48, no. 2, pp. 304-307, Feb. 2003.

[11] G. N. Nair and R. J. Evans, "Stabilization with data-rate limited feedback: Tightest attainable bounds," Systems Control Letters, vol. 41, no. 1, pp. 304-307, Sept. 2000
[12] — , "Exponential stabilisability of finite-dimensional linear systems with limited data rates," Automatica, vol. 39, no. 4, pp. 585-593, Apr. 2003.

[13] _ "Stabilization of stochastic linear systems with finite feedback data rates," SIAM Journal of Optimal Control, vol. 43, no. 2, pp. 413-436, 2004.

[14] G. N. Nair, R. J. Evans, I. M. Y. Mareels, and W. Moran, "Topological feedback entropy and nonlinear stabilization," IEEE Trans. Automat. Contr., vol. 49, no. 9, pp. 1585-1597, Sept. 2004.

[15] N. Martins, M. Dahleh, and N. Elia, "Stabilization of uncertain systems in the presence of a stochastic digital link," submitted toIEEE Trans. Automat. Contr., 2004.

[16] V. Borkar, S. Mitter, and S. Tatikonda, "Optimal sequential vector quantization of Markov sources," SIAM Journal of Optimal Control, vol. 40, no. 1, pp. 135-148, Jan 2001.

[17] S. Tatikonda, "Control under communication constraints," Ph.D. Thesis, Department of EECS, Masachussets Institute of Technology, Cambridge, MA, 2000.

[18] S. Tatikonda and S. M. Mitter, "Control under communication constraints," IEEE Trans. Automat. Contr., vol. 49, no. 7, pp. 1056-1068, July 2004.

[19] _ "Control over noisy channels," IEEE Trans. Automat. Contr. vol. 49, no. 7, pp. 1196-1201, July 2004.

[20] S. Tatikonda, A. Sahai, and S. M. Mitter, "Stochastic linear control over a communication channel," IEEE Trans. Automat. Contr., vol. 49, no. 9, pp. 1549-1561, Sept. 2004.

[21] A. S. Matveev and A. V. Savkin, "Prolem of LQG optimal control via a limited capacity communication channel," System and Control Letters, pp. 51-64, 2004.

[22] J. H. Braslavsky, R. H. Middleton, and J. S. Freudenberg, "Feedback stabilization over signal-to-noise ratio constrained channels," submitted to the IEEE transactions on automatic control, February 18, 2005.

[23] N. C. Martins and M. A. Dahleh, "Feedback control in the presence of noisy channels: "bode-like" fundamental limitations of performance," submitted to IEEE Trans. Automat. Contr., 2004.

[24] N. C. Martins, M. A. Dahleh, and J. C. Doyle, "Fundamental limitations of feedback in the presence of side information," submitted to IEEE Trans. Automat. Contr., 2005.

[25] H. S. Witsenhausen, "On information structures, feedback and causality," SIAM Journal of Control, vol. 9, no. 2, pp. 149-160, May 1971.

[26] — "Separation of estimation and control for discrete time systems," Proc. IEEE, vol. 59, no. 11, pp. 1557-1566, Nov. 1971.

[27] Y.-C. Ho, "Team decision theory and information structures," in Proceedings of the IEEE, vol. 68, no. 6, 1980, pp. 644-654.

[28] H. S. Witsenhausen, "A counterexample in stochastic optimum control," SIAM Journal of Optimal Control, vol. 6, no. 1, pp. 131-147, 1968.

[29] - "Some remark on the concept of state," in Directions in LargeScale Systems, Y. Ho and S. Mitter, Eds. Plenum, 1976, pp. 69-75.

[30] P. R. Kumar and P. Varaiya, Stochastic Systems: Estimation Identification and Adaptive Control. Prentice Hall, 1986.

[31] A. Mahajan and D. Teneketzis, "Optimal performance of feedback control systems with limited communication over noisy channels," Department of EECS, University of Michigan, Ann Arbor, MI-481092122, Control Group Report CGR-06-07, Mar. 2006. 